\title{
Immunohistochemical Detection of Hepatocellular Carcinoma in the Setting of Ongoing Necrosis after Radiofrequency Ablation
}

\author{
Tomoo Itoh, M.D., Yasuko Orba, C.T., Hidehiro Takei, M.D., Yusuke Ishida, M.D., \\ Makoto Saitoh, M.D., Hideaki Nakamura, M.D., Takashi Meguro, M.D., Shoichi Horita, M.D., \\ Miri Fujita, M.D., Kazuo Nagashima, M.D. \\ Laboratory of Molecular and Cellular Pathology (TI, YO, HT, YI, MS, KN), Hokkaido University School of \\ Medicine, Hokkaido; Hokkaido Gastroenterology Hospital (HN, TM, SH), Hokkaido; Department of \\ Pathology, Shin-Nittetsu Muroran General Hospital (MF), Muroran; and Core Research for Evolutional \\ Science and Technology (KN), Tokyo, Japan
}

\begin{abstract}
After radiofrequency ablation (RFA), hepatocellular carcinoma undergoes complete necrosis and an ongoing necrosis that is irreversible and characterized histologically by disrupted cell outlines, homogenous cytoplasmic eosinophilia, and preserved nuclear staining, with the cells appearing quite distinct from viable cancer cells. Antibody to detect singlestranded DNA (ssDNA) specifically labeled nuclei in the setting of ongoing necrosis, but not viable tumor cells, whereas human mitochondrial antibody labeled the cytoplasm of viable cells but not cells of ongoing necrosis. The results demonstrate that RFA causes denaturation of both DNA and proteins and that the immunohistochemistry of ssDNA and mitochondrial protein is useful in detection of ongoing necrosis after RFA and provides pathological information on the validity of this procedure.
\end{abstract}

KEY WORDS: Hepatocellular carcinoma, Immunohistochemistry, Mitochondria, Ongoing necrosis, Radiofrequency ablation, ssDNA.

Mod Pathol 2002;15(2):110-115

Recently, radiofrequency ablation (RFA) has been documented as a useful therapy for malignant liver tumors and in particular, hepatocellular carcinoma (1-3). Hepatic RFA causes tumor destruction in situ by thermal coagulation and protein denaturation caused by frictional heating by ionic agitation $(4,5)$. There are several significant advantages over other

Copyright (C) 2002 by The United States and Canadian Academy of Pathology, Inc.

VOL. 15, NO. 2, P. 110, 2002 Printed in the U.S.A.

Date of acceptance: October 30, 2001.

Address reprint requests to: Kazuo Nagashima, M.D., Laboratory of Molecular and Cellular Pathology, Hokkaido University School of Medicine, Kita 15, Nishi 7, Kita-ku, Sapporo 060-8638, Japan; e-mail: knagasi@med.hokudai.ac.jp; fax: 81-11-706-7806. ablation techniques, including short treatment time, a percutaneous approach after local anesthesia, the production of precise necrotic lesions, and an extremely low morbidity $(6,7)$. The effect of RFA has been pathologically verified, and this is comprised of not only complete coagulative necrosis but also a posttreatment continuous necrosis termed ongoing necrosis or incomplete necrosis. The ongoing necrosis is characterized by homogenous cytoplasmic eosinophilia, nuclear staining, and blurred cytoplasmic borders that can be distinguished from viable tumor cells by the lack of a faint basophilic substance in the cytoplasm, loss of cell borders, and a denser chromatin staining of the nuclei. However, morphological distinction between complete necrosis and ongoing necrosis is sometimes difficult, necessitating histochemical demonstration of mitochondrial enzymatic activity $(8,9)$. In search of an alternative method for detecting ongoing necrosis on paraffin specimens, we found that an antibody that detects single-stranded DNA (ssDNA) labeled the nuclei of cells in ongoing necrosis but not viable cells and that antibody to detect human mitochondria failed to label cells of ongoing necrosis in contrast to staining viable cells. The results provide useful information on the precise extent of necrosis and ongoing necrosis of hepatocellular carcinoma after FRA.

\section{MATERIALS AND METHODS}

RFA was performed using the radiofrequency (RF) generator with a 15-gauge probe that has four retractable hooks (Model 500PA; RITA Medical System, Mountain View, CA). The active needle electrode was inserted percutaneously into the tumor area under sonographic guidance. After the hook was expanded, a single application of microwaves 
was delivered at $50 \mathrm{~W}$ for 10 minutes at an average temperature of $100{ }^{\circ} \mathrm{C}$.

Assessment of treatment efficacy was evaluated by computed tomography (CT) scan and magnetic resonance (MR) imaging after the procedure. Areas of hypoattenuation that did not enhance after administration of contrast material by CT scan were considered to present necrosis, and by MR imaging, hypointense areas on unenhanced $\mathrm{T} 1$ - and T2weighted images were considered to represent necrosis.

Eight specimens from six patients who underwent biopsy or surgical resection after RFA were pathologically examined. Three patients received needle biopsy at various times after RFA, and three patients underwent surgical removal of tumors after RFA (Table 1). All specimens were fixed in neutral formalin and embedded in paraffin. Thin sections were stained with hematoxylin and eosin (HE) and with Masson trichrome. To detect denatured DNA, a polyclonal rabbit anti-ssDNA (DAKO, Glostrup, Denmark) was used at a dilution of 1: 100 (10-12). In addition, terminal deoxynucleotidyl transferase-mediated dUTP-biotin nick endlabeling (TUNEL) methods were also applied (12, 13). To detect mitochondria, mouse anti-human mitochondria monoclonal antibody MAB 1273 (Chemicon; Temecula, CA) and anti-mitochondrial antibody 113-1 (Bio Genex, San Ramon, CA) were used at dilutions of 1:150 $(14,15)$ and 1:400 $(16,17)$, respectively. Immunostaining of a mitochondrial enzyme cytochrome $c$ oxidase subunit I was also employed by using a commercially available antibody (Molecular Probes; Eugene, OR) at the dilution of 1:100 (18).

Macroscopic description of hepatocellular carcinoma followed a previously proposed classification (19). The tumors were sectioned through the largest diameter, and the areas of total necrosis, ongoing necrosis, and viable cells were estimated by image analysis (Olympus, Tokyo, Japan) and expressed as a percentage of the total tumor size.

\section{RESULTS}

The treatment efficacy was evaluated by CT scan and MR imaging after RFA therapy, and three tumors (Case 1-3) were surgically removed after 2 to 4 weeks. All tumors were roughly spherical and well demarcated, compatible with Type 1 hepatocellular carcinoma (19; Table 1). In Case 1, two nodules were operatively removed. The larger one of the two was $18 \times 12 \mathrm{~mm}$ in size at segment 4 (S4) and was ablated, whereas the smaller one, which was $5 \times 2$ $\mathrm{mm}$ in size at S6, was not treated. A significant therapeutic effect was observed by comparing ablated and nonablated tumors. In the small nonablated tumor, cancer cells consisted of a moderately well differentiated hepatocellular carcinoma and were arranged in nests with occasional intracellular bile pigments. The nuclei were larger in size and irregular in shape compared with ordinary hepatocytes; contained fine chromatin, which was arranged around the periphery; and had one or two slightly eosinophilic nucleoli. The cytoplasm was slightly basophilic, and the cell borders were clearly visible (Fig. 1A). The larger nodule that was ablated underwent coagulative necrosis, which was more prominent in the subcapsular marginal area of the tumor compared with in the central area. The tumor cells in the central area showed homogenous cytoplasmic eosinophilia with blurred cell borders (Fig. 1B). Their nuclei were diffusely stained with hematoxylin and lacked intranuclear clear spaces. These cells were considered to be in the process of ongoing necrosis or of necrobiosis in the classical sense. The marginal carcinoma cells underwent total coagulative necrosis, showing loss of cytoplasmic as well as nuclear staining (Fig. 1C).

Immunohistochemically, antibody to ssDNA failed to label normal hepatocytes and nonablated carcinoma cells (Fig. 1D) but did labele nuclei of cells in ongoing necrosis (Fig. 1E). The TUNEL method also failed to label normal as well as neoplastic viable tumor cells but labeled cells in ongoing necrosis. The number of ssDNA-labeled nuclei was identical to that of the TUNEL-positive nuclei.

TABLE 1. Clinicopathological Summary of Radiofrequency Ablation

\begin{tabular}{|c|c|c|c|c|c|c|}
\hline $\begin{array}{l}\text { Case } \\
\text { No. }\end{array}$ & Age (y), Sex & $\begin{array}{c}\text { Duration } \\
\text { after RF (w) }\end{array}$ & $\begin{array}{c}\text { Localization (Size, mm) } \\
\text { of Tumor }\end{array}$ & Necrosis & $\begin{array}{c}\text { Efficiency (\%) } \\
\text { Ongoing Necrosis }\end{array}$ & Viable \\
\hline \multicolumn{7}{|c|}{ Surgical cases } \\
\hline \multirow[t]{2}{*}{1} & $71, \mathrm{M}$ & 5.5 & $\mathrm{~S} 4(18 \times 12)$ & 70 & 30 & 0 \\
\hline & & & S6 $(5 \times 2)$-not ablated & & & 100 \\
\hline 2 & $58, \mathrm{M}$ & 2.0 & S5 $(50 \times 43)$ & 50 & 23 & 27 \\
\hline 3 & $60, \mathrm{M}$ & 2.0 & S5 $(35 \times 27)$ & 80 & 18 & 2 \\
\hline \multicolumn{7}{|c|}{ Biopsy cases } \\
\hline 4 & $40, \mathrm{M}$ & 2.0 & S4 $(18 \times 14)$ & 40 & 60 & $0^{a}$ \\
\hline 5 & $60, \mathrm{M}$ & 4.0 & S3 $(25 \times 23)$ & 70 & 30 & 0 \\
\hline \multirow[t]{2}{*}{6} & $67, \mathrm{~F}$ & 2.0 & S7 $(26 \times 26)$ & 0 & 100 & $0^{a}$ \\
\hline & & 4.0 & & 80 & 20 & $0^{a}$ \\
\hline
\end{tabular}

${ }^{a}$ Viable cells remaining were identified as nonneoplastic hepatocytes. 

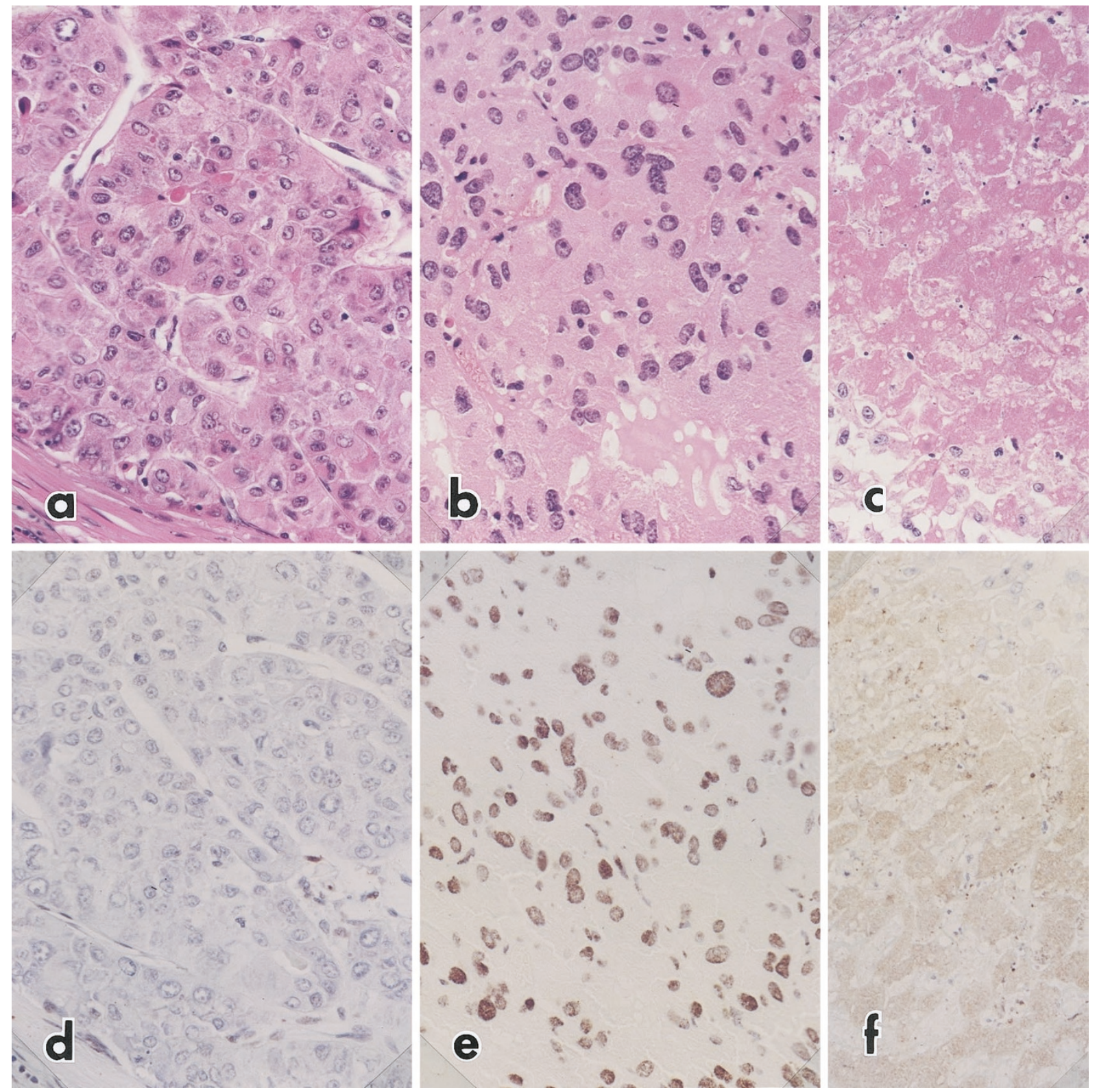

FIGURE 1. Surgically resected hepatocellular carcinoma (HCC) 4 weeks after RF therapy. A, nonablated HCC consisted of viable cells with occasional intercytoplasmic bile pigments. Hematoxylin and eosin stain (HE), 200×. B, HCC with cytoplasmic eosinophilia and intense nuclear chromatin staining was considered to be ongoing necrosis. Note the obscured cytoplasmic border. HE, 200×. C, Complete necrosis of HCC. HE, $200 \times$. D, viable HCC negative for ssDNA immunostaining; counterstained with hematoxylin. $200 \times$. E, nuclei in ongoing necrosis were labeled with ssDNA. $200 \times$. F, nuclei in complete necrosis were negative for ssDNA. $200 \times$.

Cells with complete coagulative necrosis were not stained by either method (Fig. 1F). By contrast, immunostaining using anti-human mitochondrial antibodies labeled normal and nonablated cancer cells (Fig. 2A), whereas cells in ongoing necrosis were devoid of mitochondrial staining (Fig. 2B). Areas of complete necrosis were not stained by mitochondrial staining, although the necrotic cytoplasm of a few cells contained fine granules positive for mitochondrial staining. Immunostaining of cytochrome $c$ oxidase subunit I labeled viable cells as well as some areas of cells in ongoing necrosis and complete necrosis, and this pattern of staining was different from that shown by the other two mitochondrial antibodies. Interestingly, in normal hepatic cord, mitochondrial-positive granules were located peripherally along sinusoidal space, leaving the perinuclear space empty. In cancer cells, however, mitochondrial staining was diffusely present in the cytoplasm and lacked the peripheral arrangement or the perinuclear clear space. On the basis of the results of the immunohistochemical findings, areas of complete necrosis, areas of ongoing necrosis, and viable cells were assessed in the three operated tumors (Table 1). Tumors of $<20 \mathrm{~mm}$ in diameter were totally ablated, with $70 \%$ complete 

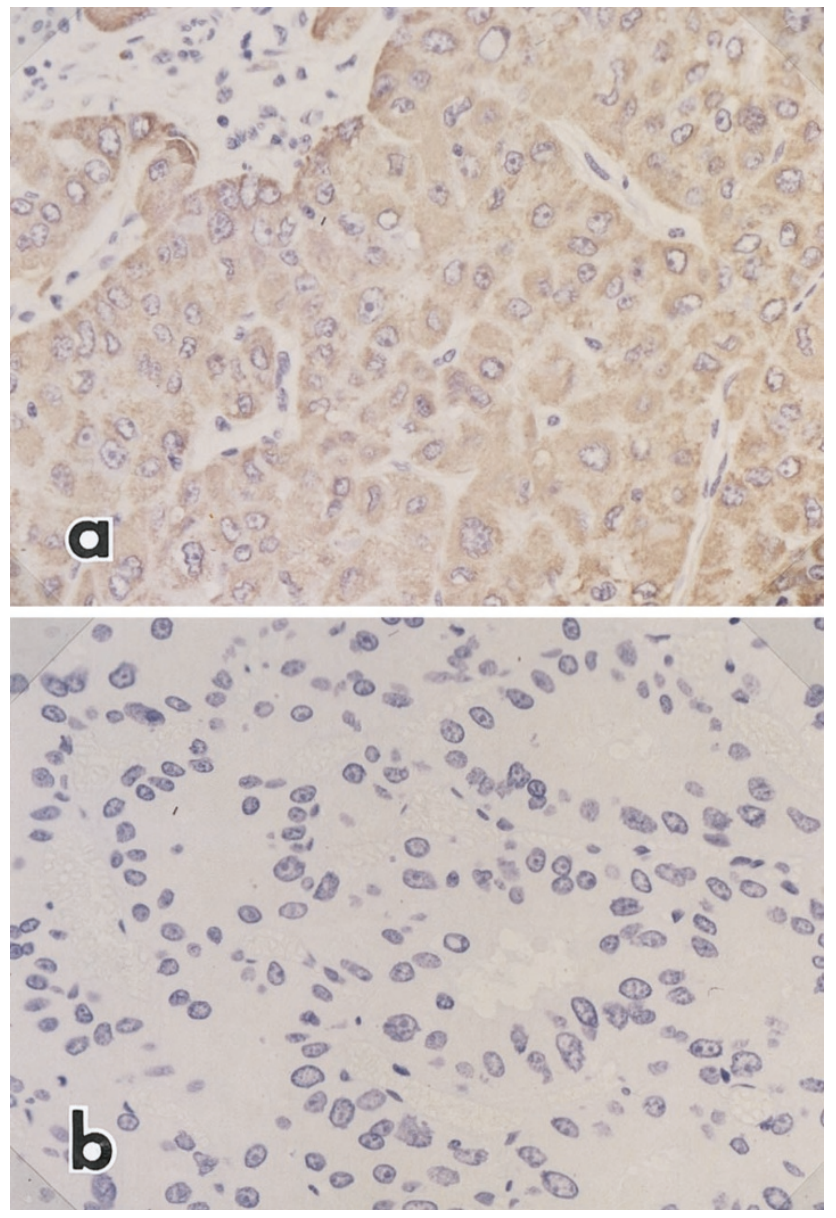

FIGURE 2. Mitochondrial staining of hepatocellular carcinoma (HCC). A, viable HCC was positive for mitochondrial immunostaining. $200 \times$. B, cytoplasm in ongoing necrosis was not stained with mitochondrial immunostaining; counterstained with hematoxylin. $200 \times$.

necrosis and $30 \%$ ongoing necrosis. In tumors of $>30 \mathrm{~mm}$ in diameter, $98 \%$ and $73 \%$ of tumors underwent complete and ongoing necrosis, leaving 2 to $27 \%$ of viable cells near the capsule, depending on the size of tumor. The total necrosis of Case 1 and $73 \%$ necrosis of Case 2 were compatible with findings estimated from CT scan and MR imaging, but $2 \%$ of the remaining viable cells in Case 3 were pathologically verified.

Four biopsy specimens from three patients (Cases 4-6) 2 to 4 weeks after RFA were evaluated by the same methods as for the surgical materials. In all materials, complete necrosis and ongoing necrosis were recognized without overt viable tumor cells by HE staining. In one case (Case 4), there was difficulty in differentiating ongoing necrosis from viable tumor cells 2 weeks after RFA by HE staining. In this material, there was an area of nonneoplastic liver tissue (Fig. 3A) that was negative for ssDNA or TUNEL but positive for mitochondrial immunostaining (Fig. 3C), although a few hepatocytes of an inner part of the specimen reacted faintly with the

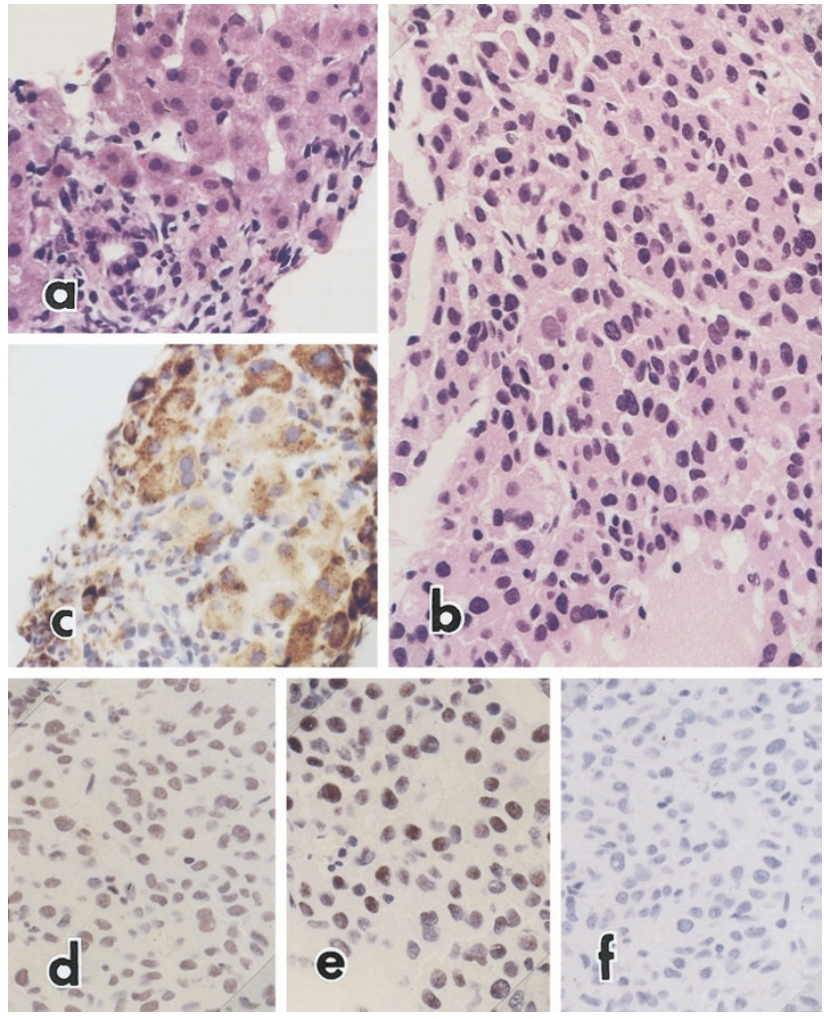

FIGURE 3. Biopsy specimen evaluated for viability of HCC 2 weeks after RF therapy. A, nonneoplastic viable hepatic tissue obtained at biopsy. HE, 200×. B, HCC with eosinophilic cytoplasmic staining, suggesting ongoing necrosis. HE, $200 \times$. C, nonablated hepatocytes shown in Figure 3A were positive for mitochondrial immunostaining, although marginal areas of the specimen stained intensely, whereas inner areas were faint. D and E, nuclei of HCC with cytoplasmic eosinophilia shown in Figure 3B were positive for both ssDNA and TUNEL, respectively. $200 \times$. F, no mitochondrial staining was observed in HCC shown in Figure 3B. $200 \times$

antibody. Neoplastic areas showed slight cytoplasmic eosinophilia with hyperchromatic nuclei (Fig. 3B) and were labeled positively with ssDNA (Fig. 3D) and TUNEL (Fig. 3E) but negatively with mitochondrial staining (Fig. 3F), which demonstrated that these cells were becoming necrotic. Areas of complete necrosis were negative for ssDNA, TUNEL, and mitochondrial proteins. In Case 6, at the first biopsy performed 2 weeks after RFA, there was $100 \%$ of ongoing necrosis but no complete necrosis. By contrast, at the second biopsy, performed 4 weeks after RFA, $80 \%$ of ongoing necrosis evolved to complete necrosis in 2 weeks, with $20 \%$ of ongoing necrosis (Table 1), confirming that the process was ongoing.

\section{DISCUSSION}

The assessment of the efficiency of therapeutic intervention in tumor treatment using newly developed procedures requires not only imaging of tumor necrosis by CT scan and MR images, but also pathological confirmation. RF energy has been ef- 
fectively used in surgery because of technical advances in the waveform modulation and in control of the output impedance and voltage by monitoring of the electrode tip temperature $(20,21)$. These advances have enabled wide application of RF current in medical and surgical treatments and particularly in the treatment of cardiac arrhythmias (22).

Since the introduction of RFA therapy for liver tumors, there have been problems in evaluating cell death by routine histological methods because of the presence of transitional features between necrosis and viable cell, classically termed necrobiosis and characterized by cytoplasmic eosinophilia and preservation of nuclear chromatin. These have been described as ghost cells (23), apoptotic-like cells (24), or electrocautery-affected cells (9). To verify cell death, electron microscopic examination (23) or demonstration of activity of mitochondrial and cytosolic enzymes has been employed $(8,9$, 24). Cryostat sections have been used for histochemical demonstration of cytochrome $c$ oxidase, succinic dehydrogenase (24), reduced nicotinamide adenine dinucleotide dehydrogenase $(8,24)$, and lactate dehydrogenase (9). To develop alternative methods to demonstrate cell viability more easily when using routine paraffin sections, we have applied an immunohistochemical technique that detects ssDNA to demonstrate denatured nuclei together with the TUNEL method. Antibodies raised against single-stranded rat liver DNA denatured by $1 \mathrm{~N}$ sodium hydroxide were shown to detect both drug-induced apoptosis and programmed cell death during embryogenesis (10). It was subsequently clarified that the antibodies recognized hexadeoxynucleotides with various base sequences (11). The apoptotic index obtained with the TUNEL method, known to detect nuclei of cells undergoing apoptosis (13), was higher than that shown by the immunohistochemistry for ssDNA, but a significant correlation was observed between the TUNEL and ssDNA (12). Applying these methods, we have now shown that nuclei of cells in necrobiosis or ongoing necrosis were labeled with ssDNA and TUNEL methods, whereas these methods failed to label viable cells. It is reasonable to detect cells in ongoing necrosis by these methods because RF causes high temperatures in the tissues (23), resulting in DNA denaturation and fragmentation with subsequent apoptosis (25). DNA denaturation caused by hyperthermia is now well accepted among pathologists who use the polymerase chain reaction technique. The process of ongoing necrosis was verified by repeated biopsy performed on Case 6 , in which $80 \%$ of all ongoing necrosis on the first biopsy became complete necrosis 2 weeks later. The necrosis observed in the RF therapy, however, may be different from that seen in myocardial infarction, because in the ablation, cell borders were lost but nuclear shapes were preserved, whereas in the infarction, cell borders were preserved, but nuclei disappeared (26). In the present studies, however, for unclear reasons, ssDNA and TUNEL occasionally labeled the background tissues and cytoplasm when applied to biopsy specimens.

A continuous high temperature $\left(100{ }^{\circ} \mathrm{C}\right.$ over 10 minutes) used in the present ablation causes cell death with protein denaturation, which has been demonstrated by the functional derangement of mitochondrial and cytoplasmic enzymes $(8,9,23)$. To test the feasibility of immunohistochemistry for mitochondrial function, we carried out mitochondrial immunostaining using two commercially available antibodies. Both antibodies labeled normal hepatocytes as well as nonablated viable hepatocellular carcinoma, but not cells in ongoing necrosis. This suggests that the mitochondria of cells in ongoing necrosis have lost antigenicity because of hyperthermia. Occasionally, however, mitochondrial staining of biopsy specimens causes marginal hyperstaining of normal hepatic cords and hypostaining especially of inner areas, probably because of uneven fixation. It was unusual for immunochemical staining of cytochrome $c$ oxidase to be positive for cells in ongoing necrosis and necrosis as well as viable cells. This suggests that the antigenic site of the enzyme may remain intact although the mitochondrial function has been lost. Thus, it is recommended that both ssDNA and mitochondrial protein immunostaining be used for detection of cells in ongoing necrosis after RA therapy.

The fact that the ablated tumor cells were in the process of ongoing irreversible necrosis may be beneficial for clinicians and patients, who can avoid unnecessary and potentially harmful overtreatment.

Acknowledgments: We are greatly appreciative of Ms. Manami Watanabe, Ms. Yukiko Fujii, Ms. Kazuko Shimizu, Ms. Mami Satoh, and Mr. Kenichi Nakase for their excellent technical assistance.

\section{REFERENCES}

1. Seki T, Wakabayashi M, Nakagawa T, Itho T, Shiro T, Kunieda $\mathrm{K}$, et al. Ultrasonically guided percutaneous microwave coagulation therapy for small hepatocellular carcinoma. Cancer 1994:74:817-25.

2. Curley SA. Radiofrequency ablation of malignant liver tumors. Oncologist 2001;6:14-23.

3. Buscarini L, Buscarini E. Therapy of HCC-radiofrequency ablation. Hepatogastroenterology 2001;48:15-9.

4. Organ L. Electrophysiologic principals of radiofrequency lesion making. Appl Neurophysiol 1976;39:69-76.

5. Huang SK. Advances in applications of radiofrequency current to catheter ablation therapy. Pacing Clin Electrophysiol 1991;14:28-42.

6. Seki T, Wakabayashi M, Nakagawa T, Imamura M, Tamai T, Nishimura A, et al. Percutaneous microwave coagulation 
therapy for patients with small hepatocellular carcinoma: comparison with percutaneous ethanol injection therapy. Cancer 1999;85:1694-702.

7. Izumi N, Asahina Y, Noguchi O, Uchihara M, Kanazawa N, Itakura J, et al. Risk factors for distant recurrence of hepatocellular carcinoma in the liver after complete coagulation by microwave or radiofrequency ablation. Cancer 2001;91: 949-56.

8. Scudamore CH, Lee SI, Patterson EJ, Buczkowski AK, July LV, Chung SW, et al. Radiofrequency ablation followed by resection of malignant liver tumors. Am J Surg 1999;177:411-7.

9. Goldberg SN, Gazelle GS, Compton CC, Mueller PR, Tanabe KK. Treatment of intrahepatic malignancy with radiofrequency ablation: radiologic-pathologic correlation. Cancer 2000;88:2452-63.

10. Naruse I, Keino H, Kawarada Y. Antibody against singlestranded DNA detects both programmed cell death and drug-induced apoptosis. Histochemistry 1994:101:73-8.

11. Kawarada Y, Miura N, Sugiyama T. Antibody against singlestranded DNA useful for detecting apoptotic cells recognizes hexadeoxynucleotides with various base sequences. J Biochem (Tokyo) 1998:123:492-8.

12. Watanabe I, Toyoda M, Okuda J, Tenjo T, Tanaka K, Yamamoto T, et al. Detection of apoptotic cells in human colorectal cancer by two different in situ methods: antibody against single-stranded DNA and terminal deoxynucleotidyl transferase-mediated dUTP-biotin nick end-labeling (TUNEL) methods. J Cancer Res 1999:90:188-93.

13. Nagashima K, Fujii Y, Tsukamoto T, Nukuzuma S, Satoh M, Fujita M, et al. Apoptotic process of cerebellar degeneration in experimental methylmercury intoxication of rats. Acta Neuropathol (Berl) 1996;91:72-7.

14. Lewis JM, Woolkalis MJ, Gerton GL, Smith RM, Jarett L, Manning DR. Subcellular distribution of the alpha subunit(s) of Gi: visualization by immunofluorescent and immunogold labeling. Cell Regul 1991;2:1097-113.

15. Gutekunst CA, Li SH, Yi H, Ferrante RJ, Li XJ, Hersch SM. The cellular and subcellular localization of huntingtin-associated protein 1 (HAP1): comparison with huntingtin in rat and human. J Neurosci 1998;18:7674-86.
16. Tickoo SK, Amin MB, Linden MD, Lee MW, Zarbo RJ. Antimitochondrial antibody (113-1) in the differential diagnosis of granular renal cell tumors. Am J Surg Pathol 1997;21:92230.

17. Michal M, Hrabal P, Skalova A. Oncocytic cystadenoma of the parotid gland with prominent signet-ring cell features. Pathol Int 1998;48:629-33.

18. Taanman JW, Burton MD, Marusich MF, Kennaway NG, Capaldi RA. Subunit specific monoclonal antibodies show different steady-state levels of various cytochrome-c oxidase subunits in chronic progressive external ophthalmoplegia. Biochim Biophys Acta 1996:1315:199-207.

19. Kanai T, Hirohashi S, Upton MP, Noguchi M, Kishi K, Makuuchi M, et al. Pathology of small hepatocellular carcinoma. A proposal for a new gross classification. Cancer 1987;60:810-9.

20. Fox JL. Experimental relationship of radiofrequency electrical current and lesion size for application to percutaneous cordotomy. J Neurosurg 1970;33:415-21.

21. Organ LW. Electrophysiologic principles of radiofrequency lesion making. Appl Neurophysiol 1976/1977;39:69-76.

22. Huang SK. Advances in applications of radiofrequency current to catheter ablation therapy. Pacing Clin Electrophysiol 1991;14:28-42.

23. Nolsoe CP, Torp-Pedersen S, Horn T, Larsen LG, Lorentzen $\mathrm{T}$, Holm HH. US-guided interstitial laser tissue ablation: "ghost cells" imply a risk of misinterpretation at follow-up biopsy [abstract]. Radiology 1995;197(P):178.

24. Solbiati L, Ierace T, Goldberg SN, Sironi S, Livraghi T, Fiocca $\mathrm{R}$, et al. Percutaneous US-guided radio-frequency tissue ablation of liver metastases: treatment and follow-up in 16 patients. Radiology 1997;202:195-203.

25. Fairbairn JJ, Khan MW, Ward KJ, Loveridge BW, Fairbairn DW, O'Neill KL. Induction of apoptotic cell DNA fragmentation in human cells after treatment with hyperthermia. Cancer Lett 1995;89:183-8.

26. Cotran RS, Kumar V, Collins T. Cellular pathology I. Robbins pathologic basis of disease. Philadelphia: Saunders; 1999. p. $16-7$.

\section{Book Review}

\section{Rosen PP: Rosen's Breast Pathology, Second Edition, 1024 pp, Philadelphia, Lippincott Williams and Wilkins, 2001 (\$299.00).}

The second edition of this comprehensive and detailed textbook is an important contribution to the literature on breast pathology and a most welcome source book for practicing pathologists. It offers expanded coverage of all aspects of breast pathology but also provides a detailed overview of anatomy, embryology, and physiology of the normal breast. Various pathologic entities are well correlated with clinical presentations of these breast diseases. It contains data not only on how to morphologically diagnose various breast conditions, but also a wealth of clinical facts that could help hospital-based pathologists to interact more efficiently with surgeons, clinical oncologists, and radiotherapists.
The book comprises 46 chapters dealing with essentially all currently known breast tumors and tumor-like conditions. All chapters are well written and profusely illustrated with excellent pictures, mostly in color. Data are presented in a readable and concise format. Very thorough discussions of laboratory handling of specimens, investigational and biomarker information, and clinicopathologic correlations are included.

This book is highly recommended to general pathologists, pathologists in training, as well as the professed pathology experts.
Asraa Namiq
Patricia Thomas
University of Kansas School of Medicine
Kansas City, Kansas 